\title{
The mysterious case of the $C$. elegans gut granule: death fluorescence, anthranilic acid and the kynurenine pathway
}

\section{Cassandra Coburn and David Gems*}

Institute of Healthy Ageing, and Department of Genetics, Evolution and Environment, University College London, London, UK

\section{Edited by:}

Elena G. Pasyukova, Institute of Molecular Genetics of the Russian Academy of Sciences, Russia

\section{Reviewed by:}

Di Chen, Nanjing University, China Shin Murakami, Touro University California, USA

Arjumand Ghazi, University of

Pittsburgh School of Medicine, USA

\section{${ }^{*}$ Correspondence:}

David Gems, Institute of Healthy Ageing, and Department of Genetics, Evolution and Environment, University College London, Gower Street, London WC1E 6BT, UK

e-mail:david.gems@ucl.ac.uk
Gut granules are lysosome-like organelles with acidic interiors that are found in large numbers within the intestine of the nematode Caenorhabditis elegans. They are particularly prominent when viewed under ultraviolet light, which causes them to emit intense blue fluorescence. Yet the function of these large and abundant organelles in this heavily-studied model organism remains unclear. One possibility is that they serve as storage organelles, for example of zinc. A new clue to gut granule function is the identification of the blue fluorescent material that they contain as a glycosylated form of anthranilic acid, which is derived from tryptophan by action of the kynurenine pathway. This compound can also serve a surprising role as a natural, endogenous marker of organismal death.

Keywords: aging, C. elegans, death fluorescence, gut granule, kynurenine, lipofuscin, organismal death, tryptophan
THE GUT GRANULE: AN ENIGMATIC NEMATODE ORGANELLE Despite decades of research on the nematode Caenorhabditis elegans, it still contains many hidden secrets. One such is the function of the prominent organelles known as gut granules, which are numerous in the intestinal cells of nematodes throughout the suborder Rhabditina (Chitwood and Chitwood, 1950). A striking feature of gut granules is the blue fluorescence that they emit under ultraviolet light (Klass, 1977; Gerstbrein et al., 2005). Clues to gut granule function include their acidic interior and capacity for endocytosis (Clokey and Jacobson, 1986; Hermann et al., 2005), both lysosome-like features (though gut granules are much bigger than normal lysosomes). This and the fluorescent material within identify gut granules as lysosome-like organelles (LROs; Hermann et al., 2005; Bernabucci et al., 2012), akin to pigmentcontaining melanosomes in mammals and eye pigment granules in Drosophila (Raposo and Marks, 2007). Thus, the identity of the blue fluorescent substance could provide a key to understanding gut granule function.

One suggestion is that the source of gut granule fluorescence is lipofuscin, a complex molecular waste production that accumulates within lysosomes in aging mammalian cells (Jung et al., 2007). Lipofuscin can contain Schiff bases, which have similar spectral similarities to the worm blue fluorescence (Fletcher et al., 1973; Klass, 1977). Consistent with this, blue fluorescence levels increase in aging worm populations (Klass, 1977; Davis et al., 1982; Gerstbrein et al., 2005). Another idea, derived from studies of C. elegans Flu mutants with altered fluorescence color and intensity, is that the blue fluorescence emanates from L-tryptophan-derived metabolites called kynurenines (Babu, 1974).

Over the years the lipofuscin interpretation has been favored (see e.g., Gill, 2006; Masse et al., 2008; Fujii et al., 2009; Jain et al., 2009; Minniti et al., 2009), perhaps because of the good fit with the theory that aging is caused by accumulation of molecular damage. Unfortunately, this interpretation (i.e., that the blue fluorescent substance is lipofuscin) is not the correct one. According to recent chemical analysis, the fluorescent substance within gut granules is a kynurenine pathway product, anthranilic acid (AA) glucosyl ester (Coburn et al., 2013), consistent with the proposal of P. Babu and S. S. Siddiqui so many years ago (Babu, 1974; Bhat and Babu, 1980; Siddiqui and Babu, 1980). This chemical identification was effected by comparing wild-type worms with glo- 1 mutants, which lack gut granules (Hermann et al., 2005). Whether or not lipofuscin exists in C. elegans remains an open question. Thus, C. elegans gut granules contain large quantities of AA. But what it is there for? Here, one may seek clues from kynurenine pathway action in mammals.

\section{THE KYNURENINE PATHWAY AND NEURODEGENERATION}

In mammals, the kynurenine pathway generates a variety of important molecules, including the co-factor nicotine adenine dinucleotide (NAD) and the neurotransmitter serotonin. Around $95 \%$ of tryptophan (the rarest essential amino acid) is consumed by this pathway (Vecsei et al., 2013). Although discovered over 150 years ago, the action of the kynurenine pathway's intermediate metabolites, known as kynurenines, has until recently been relatively little studied (Schwarcz et al., 2012). One role of kynurenines is in modulating CNS excitability (Perkins and Stone, 1982; Hilmas et al., 2001; Vecsei et al., 2013). For example, the kynurenine quinolinic acid stimulates $\mathrm{N}$-methyl-D-aspartate (NMDA) receptors (Stone and Perkins, 1981; Schwarcz et al., 2012), while kynurenic acid antagonizes all excitatory amino acid receptors.

Kynurenine pathway dysregulation has been implicated in neurological disorders, including Huntington's, Alzheimer's, and 
Parkinson's disease, multiple sclerosis, and epilepsy (Vecsei et al., 2013) as well as in neurodegeneration caused by acute insults, such as ischemia and excitotoxicity (Stone et al., 2012). Excitotoxic neurodegeneration is caused by release of high levels of excitatory neurotransmitters, which trigger an influx of calcium ions after depolarization (Rothman and Olney, 1987). Thus, calcium can act as a second messenger, triggering the initiation of necrotic cell death (Rothman and Olney, 1995). The kynurenine quinolinic acid can act as an excitotoxin: levels increase following ischemia, and correlate with increased neurodegeneration (Saito et al., 1993). Thus, one of the ways in which kynurenines may contribute to neurodegenerative disease is by inducing excitotoxic neurodegeneration.

\section{THE KYNURENINE PATHWAY IN C. elegans}

Is there a link between kynurenines and aging, particularly neurodegeneration, in $C$. elegans? Very little is known about the biology of kynurenines in nematodes. One exception relates back to gut granules: among the Flu mutants alluded to previously, altered intestinal fluorescence (Flu) phenotypes can arise from mutations affecting kynurenine pathway enzymes. For example, flu-1 mutants, which show an altered, bluish-purple gut granule fluorescence, have reduced kynurenine-3-hydroxylase activity (Siddiqui and Babu, 1980), and flu-2 mutants, which show a dull green fluorescence, have reduced kynureninase (Bhat and Babu, 1980; Figure 1A). The C. elegans genome contains homologs of genes encoding these two enzymes in the vicinity of the $f l u-1$ and flu-2 loci: a kynurenine hydroxylase, R07B7.5, and a kynureninase C15H9.7, respectively (Altschul et al., 1990; Kanehisa, 2012). Other predicted kynurenine pathway genes are also present in $C$. elegans (van der Goot and Nollen, 2013).

In Drosophila genetic and pharmacological inhibition of the kynurenine pathway enzyme tryptophan 2,3-dioxygenase (TDO) extends longevity (Oxenkrug, 2010; Oxenkrug et al., 2011). This suggests that kynurenines may contribute to pathologies of aging; however, whether this is true in $C$. elegans remains uncertain. Here RNAi knock-down of $t d o-2$ reduced the toxicity of $\alpha$-synuclein aggregation in a Parkinson's disease model, and increased lifespan (van der Goot et al., 2012). However, these effects proved to be caused by increased levels of tryptophan rather than altered levels of kynurenines (van der Goot et al., 2012; for a detailed review of the kynurenine pathway and aging

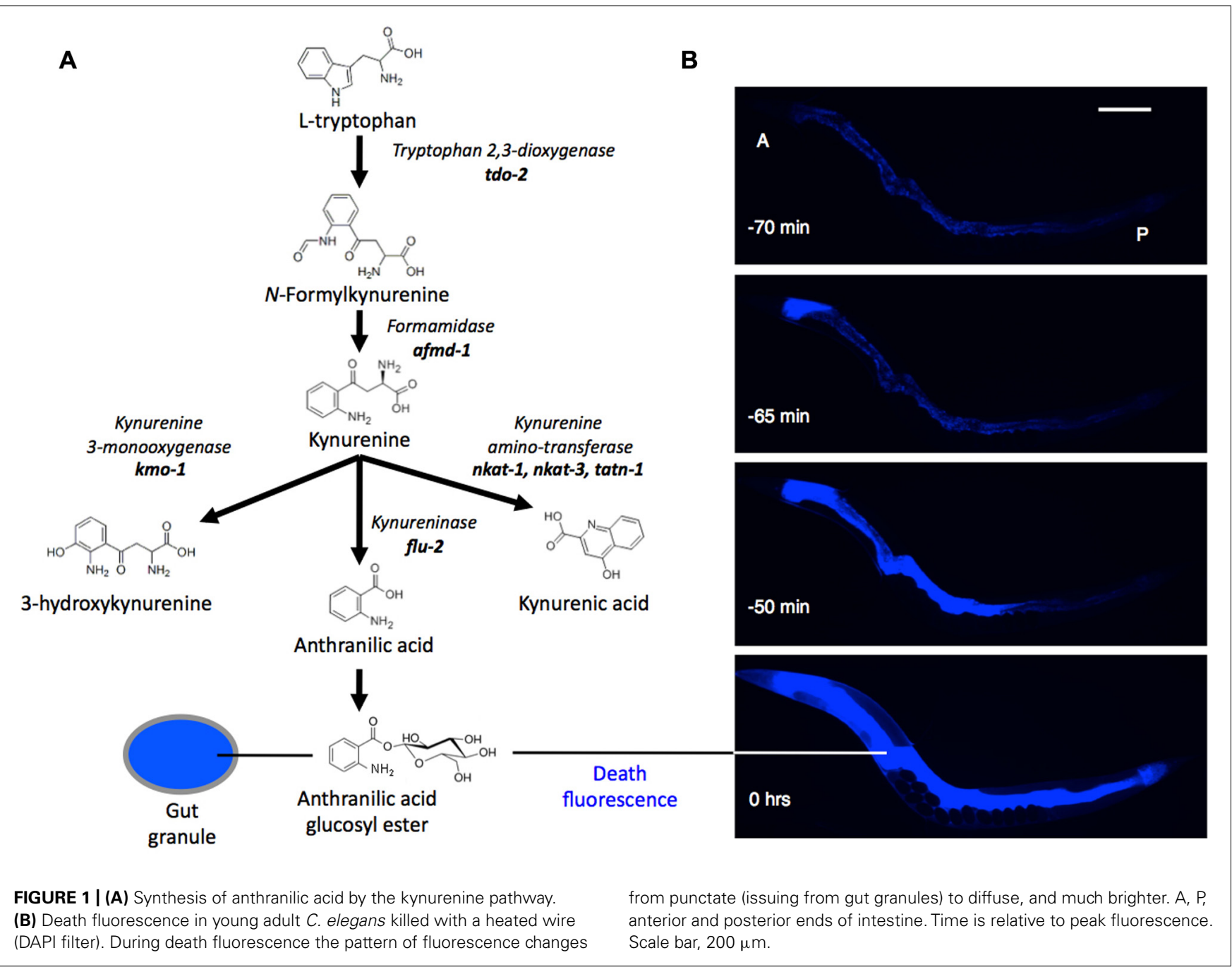


see van der Goot and Nollen, 2013). tdo-2 RNAi also abrogates gut granule fluorescence in the worm (Coburn et al., 2013).

Kynurenines also play a startling role in the biology of death in C. elegans. As they die, worms emit a dramatic burst of blue AA fluorescence (Coburn etal., 2013; Figure 1B). This death fluorescence typically occurs in an anterior to posterior wave that courses along the intestine, and is seen in both young worms subjected to lethal injury, and worms dying peacefully of old age. Death fluorescence is a somewhat eerie phenomenon in that it renders visible the passage of death through the semi-transparent body of the worm as a spectral blue glow.

Death fluorescence is promoted by the calpain-cathepsin necrotic cell death cascade. In this cascade, intracellular $\mathrm{Ca}^{2+} \mathrm{lev}-$ els rise, activating $\mathrm{Ca}^{2+}$-dependent calpains (cysteine proteases; Yamashima etal., 1996). These cause lysosomal lysis, leading to cytosolic acidosis and the destructive release of lysosomal cathepsin proteases (Yamashima and Oikawa, 2009). Mutational attenuation of this cascade often reduces death fluorescence (Coburn et al., 2013). Moreover, the intercellular propagation of death fluorescence (and, probably, necrosis) is dependent upon the innexin gap junction INX-16, reminiscent of the spread of excitotoxic neuronal death from one cell to another in mammals. How exactly the necrotic cascade leads to increased AA fluorescence remains unclear, but one possibility is that it reflects AA fluorescence dequenching as it is released from the gut granules upon organellar lysis.

\section{POSSIBLE FUNCTIONS OF ANTHRANILATES AND GUT GRANULES IN C. elegans}

The significance of AA concentrated within gut granules remains unclear. One possibility is that glycosylation of AA contributes to its accumulation; in Arabidopsis, glycosylation by UDPglucosyltransferases promotes AA accumulation by increasing compound stability (Quiel and Bender, 2003). Regarding function, one possibility is that AA serves a protective role. In mammals kynurenines can contribute to immune function (Munn et al., 1998; Fallarino et al., 2002; Piscianz et al., 2011). Moreover, AA can inhibit growth of bacterial pathogens, e.g., Legionella pneumophila (Sasaki et al., 2012). Thus, AA might have antibiotic properties in C. elegans, in which case gut granules could serve as a store of anti-bacterial agents in the event of pathogen attack. This

\section{REFERENCES}

Altschul, S. F., Gish, W., Miller, W., Myers, E. W., and Lipman, D. J. (1990). Basic local alignment search tool. J. Mol. Biol. 215, 403-410. doi: 10.1016/S0022-2836(05)80360-2

Babu, P. (1974). Biochemical genetics of Caenorhabditis elegans. Mol. Gen. Genet. 135, 39-44. doi: 10.1007/BF00433899

Bernabucci, M., Notartomaso, S., Zappulla, C., Fazio, F., Cannella, M., Motolese, M., et al. (2012). Nacetyl-cysteine causes analgesia by reinforcing the endogenous activation of type- 2 metabotropic glutamate receptors. Mol. Pain 8, 77. doi: 10.1186/1744-8069-8-77
Bhat, S. G., and Babu, P. (1980). Mutagen sensitivity of kynureninase mutants of the nematode Caenorhabditis elegans. Mol. Gen. Genet. 180 635-638. doi: 10.1007/BF00268072

Chitwood, B. G., and Chitwood, M. B. (1950). An Introduction to Nematology. Baltimore: University Park Press.

Clokey, G. V., and Jacobson, L. A. (1986). The autofluorescent "lipofuscin granules" in the intestinal cells of Caenorhabditis elegans are secondary lysosomes. Mech. Ageing Dev. 35, 79-94. doi: 10.1016/00476374(86)90068-0

Coburn, C., Allman, E., Mahanti, P., Benedetto, A., Cabreiro,

suggests a broader role for gut granules: that of chemical weapons depots for C. elegans in their war against the diverse pathogens that beset them in their natural environment (Felix and Braendle, 2010). This could also explain the presence of gut granules in the intestine, the site most likely to experience pathogenic invasion in C. elegans (Hodgkin and Partridge, 2008). Another possibility, suggested by similarities between gut granules and melanosomes, is that they are photoprotective. AA fluorescence (peak $\lambda_{\text {ex }} / \lambda_{\text {em }}$ $340 \mathrm{~nm} / 430 \mathrm{~nm}$ ) entails the conversion of damaging UV light to relatively harmless visible light, and so may protect against UV damage.

The large size of gut granules relative to ordinary lysosomes is consistent with function as a storage organelle. Moreover, gut granules are the major site of storage of zinc in the worm (Roh et al., 2012). Interestingly, when zinc levels are high, gut granule morphology changes, becoming bilobed, including an apparently non-acidic compartment in which zinc is concentrated. How distribution of zinc and AA compares in such bilobed gut granules remains to be established. It is also notable that both metal toxicity and kynurenines are determinants of neurodegenerative disease. Gut granules also stain with the lipid staining vital dye Nile red; however, results of careful analysis imply that this does not reflect the presence of lipid within gut granules (O'Rourke et al., 2009).

Ultimately, the role in C. elegans biology of gut granules and the AAs they contain remains obscure and a topic for future investigation. But we now know at least that the fluorescence of these prominent organelles issues from AA glucosyl esters, rather than lipofuscin - removing one reason for believing that worm aging is caused by accumulation of molecular damage, and opening the way for alternatively theories (Gems and de la Guardia, 2012). And we know that gut granule decay contributes to a wave of intestinal necrosis accompanied by a burst of blue anthranilate fluorescence, which serves as a useful marker for organismal death in C. elegans.

\section{ACKNOWLEDGMENTS}

We thank Alex Benedetto and our referees for comments on the manuscript. This work was supported by funding from the Biotechnology and Biological Sciences Research Council, the Wellcome Trust (Strategic Award) and the European Union (IDEAL).

F., Pincus, Z., et al. (2013). Anthranilate fluorescence marks a calcium-propagated necrotic wave that promotes organismal death in C. elegans. PLoS Biol. 11:e1001613. doi: 10.1371/journal. pbio. 1001613

Davis, B. O. Jr., Anderson, G. L., and Dusenbery, D. B. (1982). Total luminescence spectroscopy of fluorescence changes during aging in Caenorhabditis elegans. Biochemistry 21, 4089-4095. doi: 10.1021/ bi00260a027

Fallarino, F., Grohmann, U., Vacca, C., Bianchi, R., Orabona, C., Spreca A., et al. (2002). T cell apoptosis by tryptophan catabolism. Cell Death
Diff. 9, 1069-1077. doi: 10.1038/ sj.cdd. 4401073

Felix, M. A., and Braendle, C. (2010). The natural history of Caenorhabditis elegans. Curr. Biol. 20, R965-R969. doi: 10.1016/j.cub.2010.09.050

Fletcher, B. L., Dillard, C. J., and Tappel, A. L. (1973). Measurement of fluorescent lipid peroxidation products in biological systems and tissues. Anal. Biochem. 52, 1-9. doi: 10.1016/0003-2697(73) 90327-8

Fujii, M., Adachi, N., Shikatani, K., and Ayusawa, D. (2009). [FeFe]hydrogenase-like gene is involved in the regulation of sensitivity to oxygen in yeast and nematode. Genes Cells 
14, 457-468. doi: 10.1111/j.13652443.2009.01282.x

Gems, D., and de la Guardia, Y. (2012). Alternative perspectives on aging in $C$. elegans: reactive oxygen species or hyperfunction? Antioxid. Redox Signal. 19, 321-329. doi: 10.1089/ars.2012.4840

Gerstbrein, B., Stamatas, G., Kollias, N., and Driscoll, M. (2005). In vivo spectrofluorimetry reveals endogenous biomarkers that report healthspan and dietary restriction in Caenorhabditis elegans. Aging Cell 4, 127 137. doi: 10.1111/j.1474-9726.2005. 00153.x

Gill, M. S. (2006). Endocrine targets for pharmacological intervention in aging in Caenorhabditis elegans. Aging Cell 5, 23-30. doi: 10.1111/j.1474-9726.2006.00186.x

Hermann, G. J., Schroeder, L. K., Hieb, C. A., Kershner, A. M., Rabbitts, B. M., Fonarev, P., et al. (2005). Genetic analysis of lysosomal trafficking in Caenorhabditis elegans. Mol. Biol. Cell 16, 3273-3288. doi: $10.1091 / \mathrm{mbc}$. E05-01-0060

Hilmas, C., Pereira, E., Alkondon, M., Rassoulpour, A., Schwarcz, R., and Albuquerque, E. (2001). The brain metabolite kynurenic acid inhibits alpha7 nicotinic receptor activity and increases non-alpha7 nicotinic receptor expression: physiopathological implications. J. Neurosci. 21, 7463 7473.

Hodgkin, J., and Partridge, F. A. (2008). Caenorhabditis elegans meets microsporidia: the nematode killers from Paris. PLoS Biol. 6:2634-2637. doi: 10.1371/journal.pbio.1000005

Jain, C., Yun, M., Politz, S. M., and Rao, R. P. (2009). A pathogenesis assay using Saccharomyces cerevisiae and Caenorhabditis elegans reveals novel roles for yeast AP-1, Yap1, and host dual oxidase BLI3 in fungal pathogenesis. Eukaryot. Cell 8, 1218-1227. doi: 10.1128/EC. 00367-08

Jung, T., Bader, N., and Grune, T. (2007). Lipofuscin: formation, distribution, and metabolic consequences. Ann. N. Y. Acad. Sci. 1119, 97-111. doi: 10.1196/annals.1404.008

Kanehisa, L. (2012). Kyoto Encyclopedia of Genes and Genomes, $C$. elegans, Tryptophan [Online]. Available at: http://www.kegg.jp/keggbin/show_pathway?cel00380 [accessed December 27, 2012].

Klass, M. R. (1977). Aging in the nematode Caenorhabditis elegans: major biological and environmental factors influencing life span. Mech. Ageing Dev. 6, 413-429. doi: 10.1016/00476374(77)90043-4

Masse, I., Molin, L., Mouchiroud, L. Vanhems, P., Palladino, F., Billaud, M., et al. (2008). A novel role for the SMG-1 kinase in lifespan and oxidative stress resistance in Caenorhabditis elegans. PLoS ONE 3:e3354. doi: 10.1371/journal.pone.0003354

Minniti, A. N., Cataldo, R., Trigo, C., Vasquez, L., Mujica, P., Leighton, F., et al. (2009). Methionine sulfoxide reductase A expression is regulated by the DAF-16/FOXO pathway in Caenorhabditis elegans. Aging Cell 8, 690-705. doi: 10.1111/j.1474-9726. 2009.00521.x

Munn, D. H., Zhou, M., Attwood, J. T., Bondarev, I., Conway, S. J., Marshall, B., et al. (1998). Prevention of allogeneic fetal rejection by tryptophan catabolism. Science 281, 11911193. doi: 10.1126/science.281.5380. 1191

O'Rourke, E. J., Soukas, A. A., Carr, C. E., and Ruvkun, G. (2009). C. elegans major fats are stored in vesicles distinct from lysosome-related organelles. Cell Metab. 10, 430-435. doi: 10.1016/j.cmet.2009.10.002

Oxenkrug, G. F. (2010). The extended life span of Drosophila melanogaster eye-color (white and vermilion) mutants with impaired formation of kynurenine. J. Neural Transm. 117, 23-26. doi: 10.1007/s00702-0090341-7

Oxenkrug, G. F., Navrotskaya, V., Voroboyva, L., and Summergrad, P. (2011). Extension of life span of Drosophila melanogaster by the inhibitors of tryptophan-kynurenine metabolism. Fly 5, 307-309. doi: 10.4161/fly.5.4.18414

Perkins, M. N., and Stone, T. W. (1982). An iontophoretic investigation of the actions of convulsant kynurenines and their interaction with the endogenous excitant quinolinic acid. Brain Res. 247, 184-187. doi: 10.1016/0006-8993(82)91048-4

Piscianz, E., Cuzzoni, E., De Iudicibus, S., Valencic, E., Decorti, G., and Tommasini, A. (2011). Differential action of 3-hydroxyanthranilic acid on viability and activation of stimulated lymphocytes. Int. Immunopharmacol. 11, 2242-2245. doi: 10.1016/j. intimp.2011.09.009

Quiel, J. A., and Bender, J. (2003). Glucose conjugation of anthranilate by the Arabidopsis UGT74F2 glucosyltransferase is required for tryptophan mutant blue fluorescence. J. Biol. Chem. 278, 6275-6281. doi: 10.1074/jbc.M211822200

Raposo, G., and Marks, M. S. (2007). Melanosomes - dark organelles enlighten endosomal membrane transport. Nat. Rev. Mol. Cell Biol. 8, 786-797. doi: 10.1038/nrm2258

Roh, H. C., Collier, S., Guthrie, J., Robertson, J. D., and Kornfeld, K. (2012). Lysosome-related organelles in intestinal cells are a zinc storage site in C. elegans. Cell Metab.15, 88-99. doi: 10.1016/j.cmet.2011.12.003

Rothman, S., and Olney, J. (1987). Excitotoxicity and the NMDA receptor. Trends Neurosci. 10, 299-302. doi: 10.1016/0166-2236(87)90177-9

Rothman, S., and Olney, J. (1995). Excitotoxicity and the NMDA receptor - still lethal after eight years. Trends Neurosci. 18, 57-58. doi: 10.1016/0166-2236(95)93869-Y

Saito, K., Nowak, T. S. Jr., Markey, S. P., and Heyes, M. P. (1993). Mechanism of delayed increases in kynurenine pathway metabolism in damaged brain regions following transient cerebral ischemia. J. Neurochem. 60, 180-192. doi: 10.1111/j.14714159.1993.tb05836.x

Sasaki, T., Mizuguchi, S., and Honda, K. (2012). Growth inhibitory effects of anthranilic acid and its derivatives against Legionella pneumophila. J. Biosci. Bioeng. 113, 726-729. doi: 10.1016/j.jbiosc.2012.01.012

Schwarcz, R., Bruno, J. P., Muchowski, P. J., and Wu, H. Q. (2012). Kynurenines in the mammalian brain: when physiology meets pathology. Nat. Rev. Neurosci. 13, 465-477. doi: 10.1038/ nrn3257

Siddiqui, S. S., and Babu, P. (1980). Kynurenine hydroxylase mutants of the nematode Caenorhabditis elegans. Mol. Gen. Genet. 179, 21-24. doi: 10.1007/BF00268441

Stone, T. W., Forrest, C. M., Stoy, N., and Darlington, L. G. (2012). Involvement of kynurenines in Huntington's disease and stroke-induced brain damage. J. Neural Trans. 119 261-274. doi: 10.1007/s00702-0110676-8

Stone, T. W., and Perkins, M. N. (1981). Quinolinic acid: a potent endogenous excitant at amino acid receptors in CNS. Eur. J. Pharmacol. 72, 411-412. doi: 10.1016/0014-2999 (81)90587-2

van der Goot, A., and Nollen, E. (2013). Tryptophan metabolism: entering the field of aging and age-related pathologies. Trends Mol. Med. 19, 336-344. doi: 10.1016/j. molmed.2013.02.007

van der Goot, A., Zhu, W., VázquezManrique, R., Seinstra, R., Dettmer, K., Michels, H., et al. (2012). Delaying aging and the aging-associated decline in protein homeostasis by inhibition of tryptophan degradation. Proc. Natl. Acad. Sci. U.S.A. 109, 14912-14917. doi: 10.1073/pnas. 1203083109

Vecsei, L., Szalardy, L., Fulop, F., and Toldi, J. (2013). Kynurenines in the CNS: recent advances and new questions. Nat. Rev. Drug Discov. 12, 64-82. doi: 10.1038/nrd3793

Yamashima, T., and Oikawa, S. (2009). The role of lysosomal rupture in neuronal death. Prog. Neurobiol. 89, 343358. doi: 10.1016/j.pneurobio.2009. 09.003

Yamashima, T., Saido, T. C., Takita, M., Miyazawa, A., Yamano, J., Miyakawa, A., etal. (1996). Transient brain ischaemia provokes $\mathrm{Ca} 2+$, PIP2, and calpain responses prior to delayed neuronal death in monkeys. Eur. J. Neurosci. 8, 19321944. doi: 10.1111/j.1460-9568.1996. tb01337.x

Conflict of Interest Statement: The authors declare that the research was conducted in the absence of any commercial or financial relationships that could be construed as a potential conflict of interest.

Received: 30 May 2013; accepted: 21 July 2013; published online: 07 August 2013. Citation: Coburn C and Gems D (2013) The mysterious case of the C. elegans gut granule: death fluorescence, anthranilic acid and the kynurenine pathway. Front. Genet. 4:151. doi: 10.3389/fgene.2013.00151

This article was submitted to Frontiers in Genetics of Aging, a specialty of Frontiers in Genetics.

Copyright: (C) 2013 Coburn and Gems. This is an open-access article distributed under the terms of the Creative Commons Attribution License (CC BY). The use, distribution or reproduction in other forums is permitted, provided the original author(s) or licensor are credited and that the original publication in this journal is cited, in accordance with accepted academic practice. No use, distribution or reproduction is permitted which does not comply with these terms. 